\title{
Radiation by energetic electrons accelerated by wave-particle interaction: a plausible mechanism for $x$-ray emission from the Venus mantle
}

\author{
R. Bingham ${ }^{1,2}$, K. B. Quest ${ }^{3}$, V. D. Shapiro ${ }^{4}$, and B. J. Kellett ${ }^{1}$ \\ ${ }^{1}$ STFC Rutherford Appleton Lab., Space Science \& Technology Dept., Didcot, Oxon., OX11 0QX, UK \\ ${ }^{2}$ The Physics Department, University of Strathclyde, Glasgow, G4 0NG, Scotland UK \\ ${ }^{3}$ ECE Department, Univ. of California, San Diego, La Jolla, CA 92093/0407, USA \\ ${ }^{4}$ Physics Department, Univ. of California, San Diego,La Jolla, CA 92093/0319, USA
}

Received: 5 March 2007 - Revised: 15 May 2008 - Accepted: 21 May 2008 - Published: 2 July 2008

\begin{abstract}
In this paper it is argued that recently observed $\mathrm{x}$-ray emission from non-magnetic planets (Dennerl et al., 2002) can be explained as a combination of bremsstrahlung and line $\mathrm{K}$-shell radiation produced by the interaction of energetic electrons with the neutral atmosphere. Numerical simulations show that the modified two stream instability can generate energetic $100 \mathrm{eV}$ electrons that are observed and these electrons can produce $\mathrm{x}$-ray emission.
\end{abstract}

Keywords. Interplanetary physics (Plasma waves and turbulence) - Solar physics, astrophysics, and astronomy ( $\mathrm{X}$ rays and gamma rays) - Space plasma physics (Wave-particle interactions)

\section{Introduction}

On non-magnetic planets such as Mars and Venus there is no obstacle to the solar wind flow and thus, there is no planetary magnetosphere. The solar wind comes into direct contact with the planets atmosphere and the resultant coupling produces observable features and processes that are distinct from those observed at the Earth. One example is the erosion of the planetary atmosphere owing to the pickup of planetary ions by the solar wind flow (Luhmann and Bauer, 1992) and the long-term modification of the planetary water budget (Hartle and Grebowsky, 1995). A second example, and the subject of this paper, is x-ray emissions from non-magnetic planets by energetic electrons owing to unstable plasma interactions between the solar wind and planetary ions.

Correspondence to: R. Bingham

(r.bingham@rl.ac.uk)
At Venus, the Pioneer Venus Orbiter (PVO) revealed the existence of a broad $(100 \mathrm{~km})$ and turbulent dayside layer at the ionospheric boundary, where the interaction of the solar wind and the planetary ions takes place. This region is called the plasma mantle (Spenner et al., 1980). A hot electron spectrum with energies exceeding $100 \mathrm{eV}$ was observed to be typical within the Venusian mantle (Szegö et al., 1997), and quite distinct from the signatures of the solar wind and the ionosphere. Similar conclusions were drawn from measurements carried out by Viking 1 in the Martian ionosphere (Johnson and Hanson, 1991). These measurements were done using the Retarding Potential Analyzers, each of which had an upper limit of $78 \mathrm{eV}$. However, Johnson and Hanson (1991) used a novel extrapolation technique to identify an electron component more energetic than directly observed by their device. At an altitude of $850 \mathrm{~km}$ (the approximate position of the Mars mantle) a peak in the electron current was recorded. That was interpreted as an excess current due to an electron component more energetic than $78 \mathrm{eV}$, creating a secondary electron emission that was detected by the instrument. This interpretation also fits well with the direct measurement of an energetic electron distribution within the Mars mantle. The distribution measured by the Russian spacecraft Phobos 2 was observed to have a peak energy around a few hundred eV (Shutte et al., 1989).

It was also shown (Scarf et al., 1980) that a turbulent electric field is associated with the mantle. The power maximizes around frequencies of $100 \mathrm{~Hz}$, i.e. several times above the local lower hybrid frequency $\omega_{L H} \equiv \sqrt{\omega_{c p} \omega_{c e}}$, where $\omega_{c p}$ and $\omega_{c e}$ are the proton and electron gyrofrequencies, respectively.

A systematic investigation was begun to create a theoretical framework for those observations (Sagdeev et al., 1990; Szegö et al., 1991; Shapiro et al., 1995; Quest et al., 1997).

Published by Copernicus Publications on behalf of the European Geosciences Union. 




Fig. 1. A schematic of the cycloidal trajectory of a newly born ion, in the direction of the solar wind flow. The flow intersects the magnetic field (dotted line in $x-y$ plane) and creates an electric field in the z-direction.

This body of work demonstrated that the so-called modified two-stream instability (MTSI) is an expected consequence of the interaction between the counter-streaming plasma populations in the planetary mantle, with typical unstable wave frequencies several multiples above the lower hybrid frequency $\omega_{L H}$. The interaction of these waves with electrons can result in significant acceleration. In previous publications (Quest et al., 1997; Dobé et al., 1999) a hybrid code was used to simulate the processes developing in the turbulent mantle layer. Recently a kinetic Darwin code (Nielson and Lewis, 1976) has been used to simulate electron heating by waves in the mantle layer (Bryans et al., 2003). The presence of these energetic electrons may have an important macroscopically-observable consequence: their interaction with the neutral atmosphere of a planet could result in strong $\mathrm{x}$-ray emission by the mechanisms of line radiation and bremsstrahlung produced by impacts of energetic electrons with neutrals and ions (Bingham et al., 1997; Shapiro et al., 1999). The shocked solar wind that penetrates into the mantle region interacts with photo-ionized planetary gas. Photoionization is due to ultra-violet radiation from the Sun. These newly created planetary ions are picked up by the convective electric field and start to move in cycloidal trajectories, forming in the solar wind frame, a ring distribution gyrating with the transverse velocity of the solar wind and penetrating into the solar wind along the solar winds' magnetic field lines with the parallel component of this velocity (Fig. 1). The ring distribution is unstable to the generation of wave activity that is responsible for energizing the electrons. This instability is commonly referred to as the modified two stream instability (MTSI). A similar instability occurs with the interaction of the solar wind with cometary atmospheres resulting in the production of energetic $\mathrm{keV}$ electrons mea- sured by the Vega satellite (Gringauz et al., 1986; Gringauz et al., 1987) and explained by the modified two stream instability (Bingham et al., 1997; Shapiro et al., 1999).

\section{Venus x-ray emission}

In what follows we shall discuss a model of $\mathrm{x}$-ray planetary emission based on its production by energetic electrons. These electrons are accelerated inside the turbulent mantle by wave-particle interactions. They diffuse across the magnetic field, and leave the mantle at an anomalous rate provided by lower hybrid waves in the mantle. Since the lower hybrid waves are polarized almost across magnetic field lines and have frequencies much less than the electron gyrofrequency, the diffusion rate can be written as

$D=\frac{c^{2} E^{2}}{B^{2} \Delta \omega}$

Here $E \sim 100 \mathrm{mV} / \mathrm{m}$ is the wave electric field, $B \sim 10^{-4} \mathrm{G}$ is the magnetic field in the mantle, and $\Delta \omega$ is the frequency width of the wave spectrum. It follows that $D \sim 10^{16} \mathrm{~cm}^{2} / \mathrm{s}$ and the time $\tau_{D}$ needed for electrons to cross the mantle (assuming a width $\Delta \sim 100 \mathrm{~km}$ ) is

$\tau_{D} \approx \frac{\Delta^{2}}{D} \sim 10^{-2} \mathrm{~s}$

This time is quite small in comparison with the time needed for electrons to traverse along the mantle, but is comparable with the time needed for electron velocity diffusion along the magnetic field lines to $\mathrm{keV}$ energies

$\tau_{E} \approx \delta \omega \frac{k^{2}}{k_{z}^{2}} \frac{m_{e}^{2}\left(\Delta v_{z}\right)^{2}}{e^{2} E^{2}}$

Substituting $\left(\Delta v_{z}\right)^{2} \sim 2.10^{18} \mathrm{~cm}^{2} / \mathrm{s} \quad(\mathrm{keV}$ energies $)$ and $k_{z} / k \sim 10^{-1}$ yields the result $\tau_{D} \simeq \tau_{E}$. It follows that mantle electrons with $\mathrm{keV}$ energies can and will penetrate into the upper atmosphere. Since there is little or no magnetic field, they can freely precipitate down into the more dense atmosphere, where they will produce $\mathrm{x}$-ray emission in collisions with neutral atoms.

The amplitude of the transverse ion oscillations (for the evolution of the modified two stream instability only ion dynamics are important) is equal to

$a \sim \frac{e \sqrt{<E^{2}>}}{m_{i} \omega^{2}} \approx 5 \times 10^{3} \mathrm{~cm}$

This amplitude is much less than the mantle thickness, and justifies the approximation of a uniform plasma used in the present paper.

X-ray emission from Venus was first observed on 10-11 January 2001 (Dennerl et al., 2002). In X-rays, Venus is clearly detected as a half lit crescent with considerable limb brightening on the sunward side (Dennerl et al., 2002). The 
morphology of the radiation agrees well with what would be expected from both models, i.e. scattering of the energetic electrons accelerated at the dayside Venus mantle as well as scattering of the solar $\mathrm{x}$-rays by the planetary atmosphere. These data also suggest that charge exchange is not a likely source of x-ray emission.

In the pioneering paper by Dennerl et al. (2002), it was proposed that the fluorescent scattering of the solar X-rays is the sole source of the x-ray emission. Figure 2 displays the photon count rate integrated around the dayside (or nightside) at fixed distances from the Venusian center. The left vertical line is the radius of the planet, while the right line is the radius within which the majority of the photon counts were absorbed. There was a problem in that direct comparison with solar x-ray fluxes monitored simultaneously by SOHO-SEM, GOES-8 and GOES-10 spacecrafts did not show any obvious correlation with the Venus emission, but the authors argue that Venus saw a solar hemisphere which was rotated by at least 46.5 degrees from that facing the Earth. A simulation of fluorescent scattering produced (by using a standard Venusian atmospheric model) qualitative agreement with the observations. In particular, the emissions were shown to maximize in the troposphere, consistent with the Chandra observations.

Morphological arguments are not able to distinguish between the two models, i.e. if radiation is produced either by scattering of energetic electrons or solar x-rays. In both cases, the source of the radiation is located outside the scattering atmosphere. There are, however, other strong arguments that can be made in favor of energetic electrons being the source of the x-ray radiation. First, in situ balloon measurements in the terrestrial auroral ionosphere have clearly identified $\mathrm{x}$-ray emission as being dominated by energetic electrons during magnetic substorms, and not by fluorescent scattering (Anderson, 1965). Second, in situ observations have shown that non-magnetic planets are sources of $\mathrm{x}$-ray emission, where energetic electrons are theoretically expected and observationally confirmed to be present. If solar X-rays are the source of scattering outside periods of solar flare activity, why is it not observed at other planets with well developed neutral atmospheres? Finally, x-ray emission was observed from the plasma torus of Io (Clark et al., 2002), where according to Formissano et al. (1982), there is a population of energetic electrons responsible for the anomalous ionization. The Venusian emissions were observed at discrete energy levels, mainly at the Oxygen K-alpha energy at $0.53 \mathrm{keV}$, carbon K-alpha energy at $0.28 \mathrm{keV}$ and marginally from the nitrogen K-alpha energy at $0.39 \mathrm{keV}$. These results confirm earlier expectations that $\mathrm{X}$-ray satellites can analyze planetary atmospheres remotely. In the following sections we propose that bremsstrahlung and line emission from wave energized electrons present a possible alternative explanation for the bulk of the soft x-rays, demonstrating that this wellestablished process could have applications to the mantles of other unmagnetized planets.

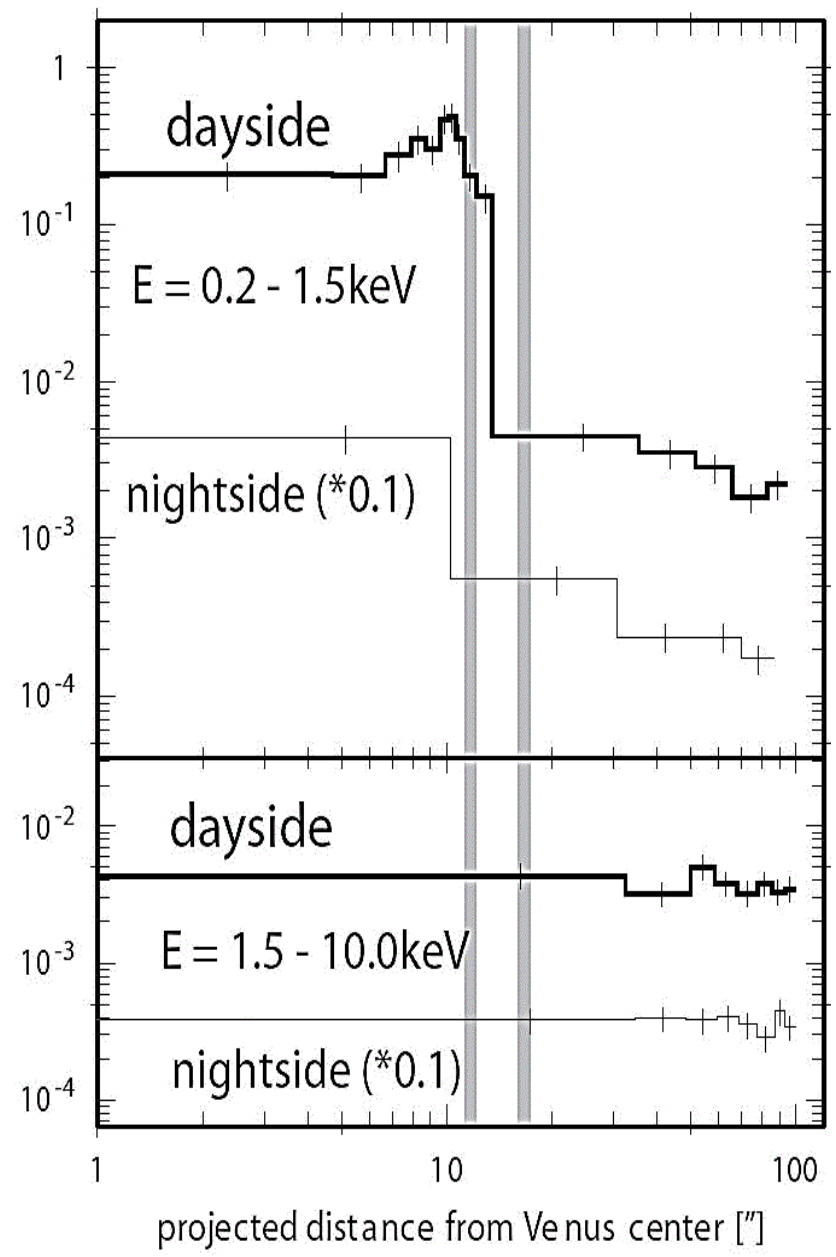

Fig. 2. Spatial distribution of photons in units of counts $\operatorname{arcmin}^{-2} \mathrm{~s}^{-1}$ around Venus in the ACIS-I observations (Dennerl et al., 2002). Two energy ranges are plotted.

\subsection{Bremsstrahlung and line radiation}

To compare a theoretical model with observations, it is necessary to estimate the total x-ray luminosity of the planet produced by both the mechanisms of bremsstrahlung and by line radiation. For electron-neutral bremsstrahlung, the power radiated by one electron in collisions with atoms $\left(\right.$ in $\mathrm{cm}^{3}$ ) can be written as

$$
P=\frac{1}{2 \pi} \int h \omega n_{N} d \sigma(\omega)
$$

where $h$ is the Planck constant, $\omega$ is the frequency, $n_{N}$ is the number density of the neutral atoms, and $d \sigma(\omega)$ is the differential cross section of bremsstrahlung given by the formula (e.g. Heitler, 1954)

$$
d \sigma(\omega)=\frac{32 \pi}{3} \frac{Z^{2} e^{2} c}{h v} r_{o}^{2} \ln \left(\pi \frac{m_{e} v^{2}}{h \omega}\right) \frac{d \omega}{\omega}
$$




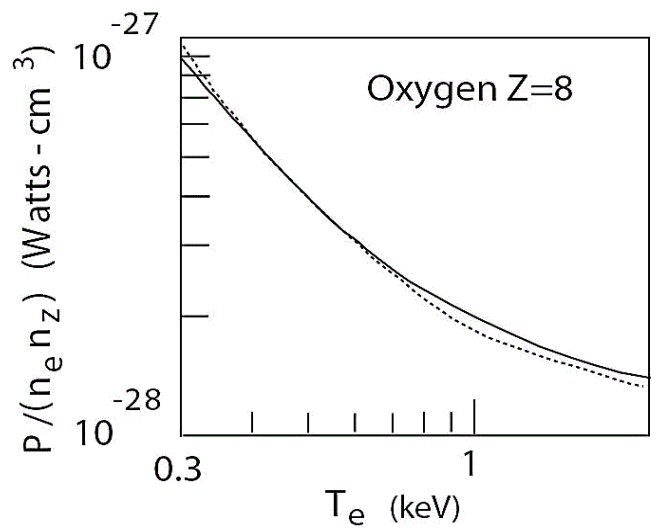

Fig. 3. Total radiation power as a function of electron energy for oxygen, from Jensen et al. (1977).

where $r_{0}=e^{2} /\left(m_{e} c^{2}\right)$ is the classical electron radius, $m_{e}$ and $v$ are the electron mass and velocity, respectively, and $Z e$ is the charge of the atomic nucleus.

The spectral density of the power radiated per unit volume and integrated over volume can be obtained from Eq. (1) by multiplying it by $f_{e}(v) d v$, where $f_{e}(v)$ is the electron distribution function. Numerical calculations described in the following section illustrate electron energization by waves in the mantle, so in accordance with these calculations we can approximate the electron distribution $f_{e}(v) \approx n_{T e} / \sqrt{2 \epsilon_{*} / m_{e}}$ as a plateau over some energy interval $\epsilon<\epsilon_{*}$, where $\epsilon_{*}$ is the upper energy bound of the electron distribution, and $n_{T}$ is the total number density of the energetic electrons. To estimate the radiation with radius $R$ and thickness $\Delta R$ it is necessary to multiply the result over the volume $2 \pi R^{2} \Delta R$. Substituting numerical values for the physical constants, the differential spectral density of the bremsstrahlung radiation is

$d L_{B R}=6.2 \times 10^{-40} Z^{2} R^{2} \frac{n_{T e}}{\sqrt{\epsilon_{*}(e V)}} \ln \left(2 \pi \frac{\epsilon_{*}}{h \omega}\right)$

The total bremsstrahlung radiation produced by the electron neutral collisions can be easily obtained from Eq. (3) by integrating over all radiated frequencies $\omega \leq 2 \pi \epsilon_{*} / h$. The result can be written as

$L_{B R}=6 \pi \cdot 10^{-25} n_{0} n_{T E} R^{2} \Delta R \sqrt{\epsilon_{e}(\mathrm{eV})}(\mathrm{ergs} / \mathrm{s})$

The scattered radiation at Venus was observed to maximize at approximately $140 \mathrm{~km}$ above the planetary surface (Dennerl et al., 2002). Substituting typical planetary parameters, $n_{0}=10^{9} \mathrm{~cm}^{-3}, n_{T E}=10 \mathrm{~cm}^{-3}, \epsilon_{e}=100 \mathrm{eV}, R \simeq 10^{9} \mathrm{~cm}$, and $\Delta R=10^{7} \mathrm{~cm}$, into Eq. (4) above, we estimate that the total luminosity produced by bremsstrahlung responsible for the continuous part of the spectrum is quite low, $L_{B R}=3.10^{11} \mathrm{erg} / \mathrm{s}$.

In addition to bremsstrahlung, energetic electrons (in hundreds of $\mathrm{eV}$ to the $\mathrm{keV}$ energy range) are responsible for $\mathrm{K}$ shell line radiation from partially ionized and neutral atoms whose bound electrons are excited in inelastic collisions with these suprathermal electrons. The bound electrons quickly de-excite and re-radiate energy they obtained in the collisions. The detailed numerical calculations of the line radiation (also known in nuclear fusion community as impurity radiation) has been done by Jensen et al. (1977). The basic physics of radiation, however, can be explained using a simple picture proposed by Dawson (1981). He assumed that the cross section of this process is determined by inelastic electron-electron collisions, having a small impact parameter and thereby leading to relatively large angle scattering of the colliding electrons. The Coulomb logarithm, which usually increases the collisional cross section by a factor 10 to 20 , owing to the input of distant collisions, is in this case (of line radiation) a strong function of the factor $\mathrm{Z}$ and is of the order of one or less than one. Consequently the radiated energy production rate can be written as

$P=5 \times 10^{-18} \Gamma \frac{n_{0} n_{T e}}{\sqrt{\epsilon}}$

in units of ergs per cubic centimeter per second. The numerical factor $\Gamma$ appears here instead of the Coulomb logarithm and for oxygen atoms is equal to 0.1. In Fig. 3, taken from Jensen et al. (1977), the photon energy production rate for oxygen atoms is shown as a function of electron energy. Also shown by a dotted line is the asymptotic value (Eq. 5). It follows from the figure starting from electron energies $\epsilon \approx 200 \mathrm{eV}$, this asymptote fits well with the more accurate numerical results of Jensen et al. (1977). We have integrated $P$ over the volume where the radiation is produced, and over all electron velocities, assuming (as was done above) that the energetic electrons have a plateau type distribution function over velocities. The result for the total radiation per unit time can be written as

$L_{\text {line }} \approx 2 \pi \cdot 10^{-17} \Gamma n_{0} n_{T e} R^{2} \Delta R \frac{1}{\sqrt{\epsilon_{e}(\mathrm{eV})}}(\mathrm{ergs} / \mathrm{s})$

These calculations show that the total luminosity in line radiation for typical planetary parameters exceeds that of bremsstrahlung by several orders of magnitude, $L_{\text {line }} \approx 2.10^{16} \mathrm{ergs} / \mathrm{s}$. These computed luminosities are comparable with the typical luminosity in the cometary case, and they are well above the Chandra observational limits. We therefore can predict that a planetary $\mathrm{x}$-ray spectrum must be discrete, and reflect the relative abundances of components of the planetary atmosphere.

\section{Simulation results}

The physical processes in the dayside mantle are governed by the MTSI, as discussed in the introduction. In Dobé et al. (1999) it was shown that the fastest developing mode of the MTSI, (which is a better match to the observational PVO data) is generated by the interaction between the ionospheric oxygen ions and the cold electron beam, created 
through pick-up by the solar wind flow. Electron pick-up takes place over a few electron gyroperiods, or essentially instantaneously. Although the cold electron beam has little kinetic energy of its own, the wave particle resonance is sustained for a longer time owing to electron pick-up under the combined action of the magnetic field and the convective electric field of the solar wind. This makes possible a substantial transfer of energy from the solar wind flow to the waves. The excited waves are predominantly electrostatic, and propagate over a wide range of angles with respect to the ambient magnetic field. It is significant that the excited waves are able to couple planetary ions to the solar wind flow almost immediately, beginning at the linear stage of instability. The most unstable mode corresponds to the resonance condition, where the Doppler shifted frequency coincides with the frequency of the whistler mode. This resonance is similar to the condition of the well-known Buneman instability (Buneman, 1959) in an unmagnetized plasma, where the Doppler-shifted frequency coincides with the Langmuir frequency.

The dispersion relation describing the MTSI wave excitation can be written as

$$
\begin{aligned}
& \frac{\omega_{p e}^{2}}{\omega_{c e}^{2}}\left(1+\frac{\omega_{p e}^{2}}{k^{2} c^{2}}\right)-\frac{\omega_{p e}^{2}}{\left(\omega-k u_{e}\right)^{2}} \frac{k_{\|}^{2} c^{2}}{k^{2} c^{2}+\omega_{p e}^{2}} \\
& \quad=\frac{Z^{\prime}\left(\omega_{p}\right)}{2 k^{2} D_{p}^{2}}+\frac{Z^{\prime}\left(\omega_{p i}\right)}{2 k^{2} D_{i}^{2}}
\end{aligned}
$$

In Eq. (6) and in the numerical analysis to be presented below we assume the presence of only one component of cold fluid electrons. Both solar wind protons and heavy (planetary) ions are treated kinetically. We define $\omega_{p}=\left(\omega-k u_{p}\right) / k v_{T p}$, $\omega_{i}=\omega / k v_{T i}, D_{p}^{2}=T_{p}\left(4 \pi e^{2} n_{0 p}\right), D_{i}^{2}=T_{i} /\left(4 \pi e^{2} n_{0 i}\right)$, $v_{T i, p}^{2} / m_{i, p}$, and $u$ is the solar wind velocity. The plasma dispersion function (Fried and Conte, 1961) is defined as

$$
Z(z)=\int_{-\infty}^{\infty} d \zeta \frac{\exp \left(-\zeta^{2}\right)}{\zeta-z}
$$

and $Z^{\prime}(z) \equiv d Z(z) / d z$ denotes the first derivative with respect to the total argument. The wave frequency satisfies $\omega_{c p} \ll \omega \ll \omega_{c e}$, where $\omega_{c p}$ and $\omega_{c e}$, are the proton and electron gyrofrequencies, respectively. It follows that electrons are magnetized in these oscillations, while protons and ions are not. As discussed above, the most unstable mode corresponds to the case when the Doppler shifted frequency $\omega-k u$ coincides with the frequency of the whistler mode. Thus, the resonance condition can be written as

$k u_{e}=\omega_{c e} \frac{k_{\|} k c^{2}}{k^{2} c^{2}+\omega_{p e}^{2}}$

where we assume $\omega \ll k u_{e}$.

In Fig. 4 the growth rate of the most unstable mode satisfying the resonance condition is plotted as a function of the mode frequency. The solid line is the analytical solution

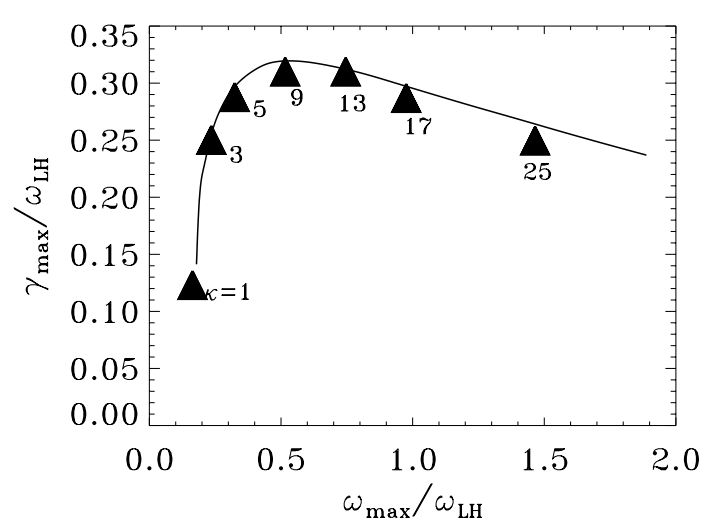

Fig. 4. Relationship between the maximum linear growth rate and the corresponding real frequencies for different $k$ values (Dobé et al., 1999).

of the dispersion relation (Eq. 6). A parameter that changes along the curve is $\kappa=\left(k_{\|} / k\right) \sqrt{m_{p} / m_{e}}$, the angle at which the wave is propagating with respect to the ambient magnetic field: $k_{\|}$is the component of the wave vector $k$ parallel to $B$. The variables $m_{p}$ and $m_{e}$ are the proton and electron masses, respectively. Triangles in the figure show the values of the frequency and the growth rate of the most unstable mode obtained in the numerical simulation for the different values of $k$ listed in the figure (Dobé et al., 1999). The analytical and numerical results are seen to be in good agreement. It also can be seen from the figure that excited waves have frequencies around the lower hybrid frequency, and a growth rate comparable with the frequency. Excitation of waves is possible over a broad range of angles $k_{\|}<0.5 k$.

The saturated wave energy varies in the range of a few percent up to $25 \%$ of the solar wind kinetic energy. The wave energy can be approximated by the formula

$W=\sum_{k} \omega_{k} \frac{\partial D}{\partial \omega_{k}} \frac{\left|E_{k}\right|^{2}}{8 \pi}=\frac{\omega}{k u_{e}} \frac{\omega_{p e}^{2}}{\omega_{c e}^{2}} \frac{E^{2}}{4 \pi}$

where $D$ is the linear dispersion relation written in a form that satisfies $D=0$, and where we assume $\omega_{p e}^{2} / \omega_{c e}^{2}=3.10^{3}$. As explained, the free energy source for instability is in the protons of the solar wind, and therefore the saturated level of the wave energy is comparable with solar wind kinetic energy. The average amplitude of the oscillating electric field $\sqrt{|E|^{2}}$ can be estimated as $10-50 \mathrm{mV} / \mathrm{m}$, in agreement with PVO observations.

We note here that for the large level of the excited wave energy, the electron acceleration by waves starts to become important. The wave frequency is of the order of $\omega_{L H}$, i.e. it is between the electron and proton gyrofrequencies. Electrons are magnetized in these oscillations, and the condition for the wave-particle resonance takes the form $\omega=k_{\|} v_{\| e}$. At the same time ions and protons are not magnetized in these oscillations, and thus the wave-particle condition is $\omega=k_{\perp} v_{\perp(i, e)}$. 

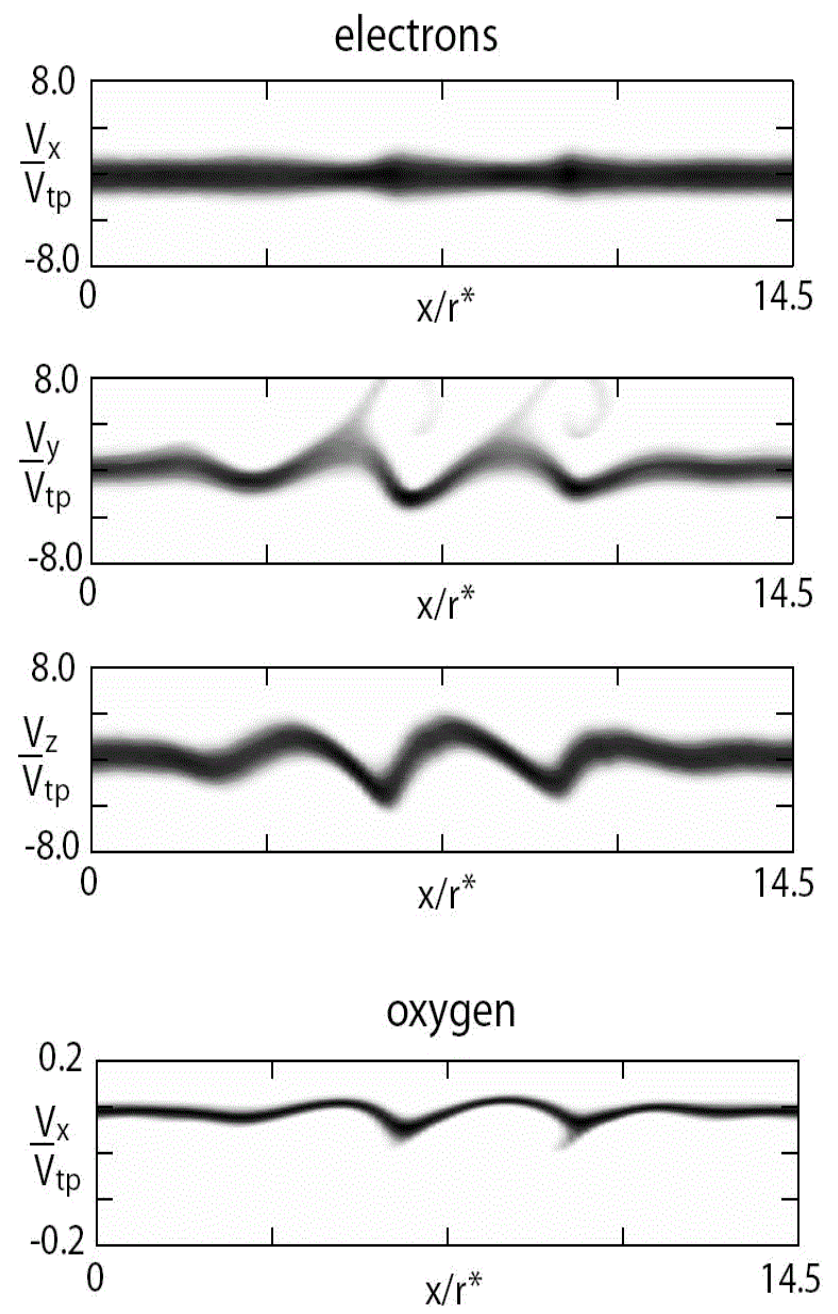

Fig. 5. Electron and ion phase space normalized to the initial proton thermal velocity at the saturation of the linear stage of the PIC simulation.

The lower hybrid waves propagate predominantly at right angles to the magnetic field $\left(k_{\|} \ll k_{\perp}\right)$, so that their phase velocity along magnetic field exceeds significantly the phase velocity across the field. Therefore these waves are able to be in simultaneous phase resonance with both fast but magnetized electrons and slow unmagnetized protons. In our case these waves are transferring the energy from the heavy energetic proton component of the solar wind to the cold planetary electrons, leading to their energization. Electron acceleration by waves is crucial for our model of $\mathrm{x}$-ray emission, since in this model emission is produced by impacts of energetic electrons with neutrals and ions.

In Figs. 5 and 6 we show the results from a simulation using a standard one-dimensional particle-in-cell Darwin simulation code (Nielson and Lewis, 1976). As in the case of those simulations, we assume a hot solar wind species streaming through an (initially) stationary cold ion species. The electrons are relatively cold at the start of the run and
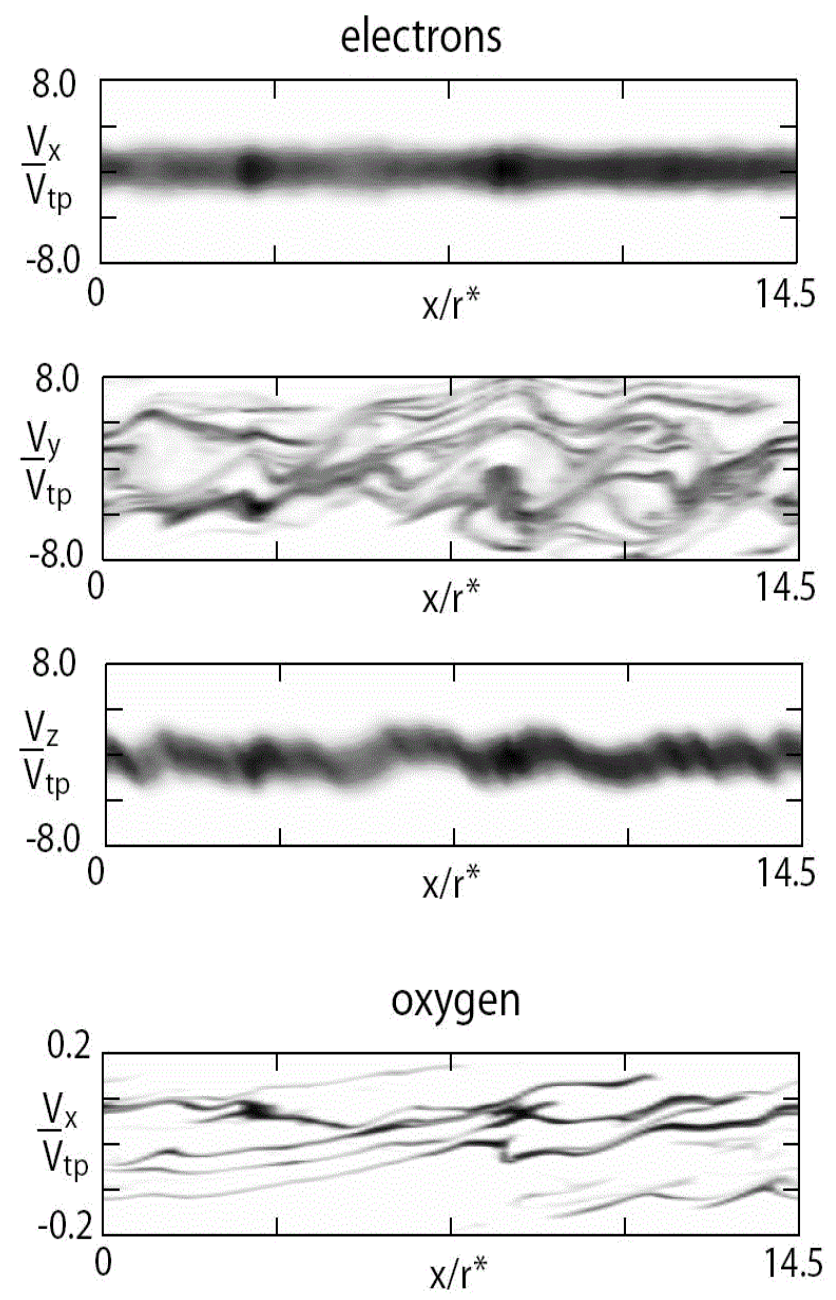

Fig. 6. Electron and ion phase space normalized to the initial proton thermal velocity at the well developed turbulent phase of the PIC simulation.

by current neutrality, drift at a speed $u_{e}=\left(n_{0 p} u /\left(n_{0 i}+n_{0 p}\right)\right)$ intermediate between the ions and protons. A mass ratio of $m_{p} / m_{e}=400$ is assumed. The ambient magnetic field lies in the x-y plane such that $B_{x} \ll B_{y}$. In Fig. 5 we display the phase space of the electrons and ions at a time close to wave saturation. The MTSI is excited to a nonlinear state, and the behavior of the ions and protons (not shown) is similar to the earlier, hybrid runs. Wave saturation amplitudes are likewise similar to those obtained earlier. As expected, however, the electrons can now be seen to form vortices in the fieldaligned (effectively $y$ ) direction.

In Fig. 6 the phase space is displayed for the postsaturation (turbulent) phase. The ions have been significantly accelerated as before, and the electrons have likewise phasemixed to an energy that is between 10-20 times their initial thermal energy. Using parameters believed typical for Venus, this would result in a fraction of the electrons being accelerated to energies above $100 \mathrm{eV}$, consistent with observations. 
Further, interaction of such electrons with the neutral atmosphere could be a potential source of soft x-ray emission described in the previous section.

\section{Conclusions}

We have shown through a combination of theory, experiment, and simulation that the fluid like branch of the MTSI, driven by the pickup of the planetary electrons by the solar wind, can strongly couple the solar wind flow to the ionospheric plasma in both energy and momentum. It is responsible for the creation of a turbulent mantle area at the ionospheric boundary where wave-particle interaction results in strong electron heating. Energetic electrons are precipitating from the mantle down to the regions of dense planetary atmosphere. Colliding with the neutral planetary atmosphere the energetic electrons are producing $\mathrm{x}$-ray emission by a combination of bremsstrahlung and $\mathrm{K}$-shell line radiation. In the future, multi-dimensional simulations including neutral collisions should be performed in order to obtain a more realistic model of the acceleration process. Codes such as ADAS can then be used to both test the simulation output as well as make theoretical detailed predictions concerning the expected soft $\mathrm{x}$-ray spectra. Finally, these predictions can be tested by future observations of x-rays at other planetary bodies, and may lead to extrasolar planet atmospheric composition using very large $\mathrm{x}$-ray detectors mounted say on the Moon.

\section{Appendix A}

There are two alternative approaches for the problem of wave-particle interaction. In one case the particle is interacting with waves having regular phase. For effective interaction the particle velocity must be equal to the velocity with which the wave phase is propagating in space, as it takes place e.g. in linear accelerators. An opposite case is realized in the turbulent plasma, where waves have random phases. Interaction with waves in the random phase approximation (RPA) results in a stochastic particle walk in velocity space, i.e. in velocity diffusion. Let us consider the last case in more detail; the wave electric field in that case can be represented in a form

$E_{z}=\frac{1}{2} \sum_{k} E_{k z} \exp i\left(k z-\omega t+\psi_{k}\right)+c . c$.

where $\psi_{k}=k z(t=0)+\varphi_{k}$ is the random phase of wave harmonic, $\varphi_{k}$ is the initial value of that phase. Then from the equation of particle motion along the electric field

$m_{e} \frac{d \nu_{z}}{d t}=-e E_{z}$ we have

$v_{z}=-\frac{e}{2 m_{e}} \sum_{k z} \frac{\exp i\left(k z-\omega t+\psi_{k}\right)}{i(k v-\omega)}+c . c$.

where in the integration of the r.h.s. of Eq. (A3) we neglected the nonlinear evolution of particle velocity $v$. Multiplying the r.h.s. Eqs. (A2) and (A3) and averaging over random wave phases (random phase approximation, RPA) we obtain the following relationship for the rate of velocity diffusion in the RPA

$$
\begin{gathered}
\frac{d}{d t} v_{z}^{2}-2 \pi \frac{e^{2}}{m_{e}^{2}} \sum_{k>0}\left|E_{z k}\right|^{2} \delta(k v-\omega) \\
=\frac{e^{2}}{m_{e}^{2}} \frac{\left|E_{k z}\right|^{2}(k=\omega / v)}{v}
\end{gathered}
$$

where we have assumed that the wave spectrum is continuous and changed $\sum_{k} \rightarrow \frac{1}{2 \pi} \int d k$. The r.h.s. of Eq. (A4) is the square of velocity (energy) diffusion coefficient. It can be substituted in the form $D_{E}=\left(\Delta v_{z}^{2}\right) / \tau_{E}$. Using expression (A4) for the diffusion coefficient and relationships $\left|E_{z k}\right|^{2}=\frac{\left|E_{z}\right|^{2}}{\Delta k}$ and $\Delta \omega=\Delta k v$ it is easy to obtain the equation for $\tau_{E}$ the typical time of velocity diffusion created by waves.

Acknowledgements. We would like to acknowledge the many invaluable contributions made by Karoly Szegö and Zoltan Dobé during our years of fruitful collaboration. This work was partially supported by NASA grant NAG5-11754 as well as CfFP the Centre for Fundamental Physics (CfFP) at the Rutherford Appleton Laboratory.

Topical Editor R. Forsyth thanks two anonymous referees for their help in evaluating this paper.

\section{References}

Anderson, K. A.: Baloon measurements of x-rays in auroral zone, in: Auroral Phenomena, edited by: Walt, M., page 46, Stanford Univ. Press, California, 1965.

Bingham, R., Dawson, J. M., Shapiro, V. D., Mendis, D. A., and Kellet, J.: Generation of x-rays for comet Huykatake, Science, 275, 49-51, 1997.

Bryans, P., Quest, K. B., Shapiro, V. D., Bingham, R., and Tourner, M.: X-ray emission from planets Venus and Mars: theoretical model and numerical simulations, EOS Trans. AGU, 84(46), Fall Meet. Suppl. Abstract SM31C-1122, 2003.

Buneman, O.: Dissipation currents in ionized media, Phys. Rev., 115, 503-517, 1959.

Clark, J. T., Ajello, J., Ballester, G., et al.: Ultraviolet emission from magnetic footprints of Io, Ganymede and Europe on Jupiter, Nature, 415, 997-999, 2002.

Dawson, J. M.: Advanced Fusion Reactors, in: Fusion, part B, edited by: Teller, E., page 465, Academic, San Diego, 1981.

Dennerl, K., Burwitz, V., Englhauser, J., Lisse, C., and Wolk, S.: The discovery of X-rays from Venus with Chandra, Astronomy and Astrophysics, 386, 319-330, 2002. 
Dobé, Z., Quest, K. B., Shapiro, V. D., Szegö, K., et al.: Interaction of the solar wind with unmagnetized planets, Phys. Rev. Lett., 83, 260, 1999.

Fried, B. D. and Conte, S. D.: The Plasma Dispersion Function, Academic Press, New York, 1961.

Formissano, V., Galeev, A. A., and Sagdeev, R. Z.: The role of the critical ionization velocity phenomena in the production of inner coma cometary plasma, Planet. Space Sci., 30, 491-497, 1982.

Gringauz, K. I., Gombosi, T. I., Remizov, A. P., Apathy, I., Szemerey, I., Verigin, M. I., Denchikova, L. I., Dyachkov, A. V., Keppler, E., Klimenko, I. N., Richter, A. K., Somogyi, A. J., Szego, K., Szendro, S., Tatrallyay, M., Varga, A., and Vladimirova, G. A.: First in situ plasma and neutral gas measurements at comet Halley, Nature, 321, 282-285, 1986.

Gringauz, K. I., Remizov, A. P., Verigin, M. I., Richter, A. K., Tàtrallyay, M., Szegö, K., Klimenko, I. N., Apàthy, I., Gambosi, T. I., and Szemerey, T.: Analysis of the electron measurements from Plasmag-1 experiment on board Vega 2 in the vicinity of comet P/Halley, Astron. Astrophys., 187, 287-289, 1987.

Hartle, R. E. and Grebowsky, J. M.: Planetary loss from light ion escape on Venus, Adv. Space Res., 15, 117-122, 1995.

Heitler, W.: The Quantum Theory of Radiation, Valley Books, Cupertino, California, 1954.

Jensen, R. V., Post, D. E., Grasberger, W. H., Tarter, C. B., and Lokke, W. A.: Calculations of impurity radiation and its effects on Tokamak experiments, Nucl. Fusion, 17, 1187-1196, 1977.

Johnson, F. S. and Hanson, W. B.: Viking 1 electron observations at Mars, J. Geophys. Res., 96, 11 097-11 118, 1991.

Luhmann, J. G. and Bauer, S. J.: Solar wind effects on atmosphere evolution at Venus and Mars, in Venus and Mars: Atmospheres, ionospheres, and solar wind interactions; Proceedings of the Chapman Conference, Balatonfured, Hungary, 4-8 June, 417-430, American Geophysical Union, Washington D.C., 1992.

Nielson, C. W. and Lewis, H. R.: Particle code models in the nonradiative limit, in: Methods of Computational Physics, edited by: Alder, B., Fernbach, S., and Rotenberg, M., 16, 367, Academic, New York, 1976.
Quest, K. B., Shapiro, V., K. Szegö, and Dobé, Z.: Numerical simulation of the lower hybrid streaming instability: consequences for the Venus mantle, Geophys. Res. Lett., 24, 301-304, 1997.

Sagdeev, R. Z., Shapiro, V. D., Shevchenko, V. I., Zacharov, A., Kiraly, P., Szegö, K., Nagy, A. F., and Grard, R. J. L.: Wave activity in the neighborhood of the bow shock of Mars, Geophys. Res. Lett., 17, 893-896, 1990.

Scarf, F. L., Taylor, W. W. L., Russell, C. T., and Elphic, R. C.: Pioneer Venus plasma wave observations - The solar-wind-Venus interaction, J. Geophys. Res., 85, 7599-7612, 1980.

Shapiro, V. D., Szegö, K., Ride, S. K., Nagy, A. F., and Shevchenko, V. I.: On the interaction between shocked solar wind and the planetary ions in the dayside of Venus, J. Geophys. Res., 100, 21 289-21 306, 1995.

Shapiro V. D., Bingham, R., Dawson, J. M., Dobé, Z., Kellet, B. J., and Mendis, D. A.: Energetic electrons produced by lower hybrid waves in the cometary environment and soft x-ray emission: bremsstrahlung and line k-shell radiation, J. Geophys. Res., 104, 2537-2554, 1999.

Shutte, N. M., Diachkov, A. V., Kiraly, P., P., Cravens, T. T., Gombosi, T. I.: Observation of electron and ion fluxes in the vicinity of Mars with the HARP spectrometer, Nature, 341, 614-616, 1989.

Spenner, K., Knudsen, W. C., Miller, K. L., Novak, V., Russell, C. T., and Elphic, R. C.: Observations of the Venus mantle, the boundary layer between solar wind and ionosphere, J. Geophys. Res., 85, 7655-7662, 1980.

Szegö, K., Shapiro, V. D., Sagdeev, R. Z., Shevchenko, V. I., Kasprzak, W. T., and Nagy, A. F.: Physical processes in the plasma mantle of Venus, Geophys. Res. Lett., 18, 2305-2308, 1991.

Szegö, K., Dobé, Z., Knudsen, W. C., Nagy, A. F., and Shapiro, V. D.: Energetic electrons in the dayside mantle of Venus, J. Geophys. Res., 102, 2175-2184, 1997. 